\title{
Should we use cortical bone screws for cortical bone trajectory?
}

\author{
Masaki Ueno, MD, PhD, ${ }^{1}$ Rina Sakai, PhD, ${ }^{2}$ Kensei Tanaka, MA, ${ }^{2}$ Gen Inoue, MD, PhD, ${ }^{1}$ \\ Kentaro Uchida, PhD, ${ }^{1}$ Takayuki Imura, MD, ${ }^{1}$ Wataru Saito, MD,1 Toshiyuki Nakazawa, MD, PhD, ${ }^{1}$ \\ Naonobu Takahira, MD, PhD, ${ }^{1,3}$ Kiyoshi Mabuchi, PhD, ${ }^{2}$ and Masashi Takaso, MD, PhD'
}

\begin{abstract}
1Department of Orthopaedic Surgery, Kitasato University School of Medicine; and Departments of ${ }^{2 B i o m e d i c a l ~ E n g i n e e r i n g ~ a n d ~}$ ${ }^{3}$ Rehabilitation, Kitasato University School of Allied Health Sciences, Sagamihara, Kanagawa, Japan
\end{abstract}

\begin{abstract}
OBJECT In 2009, Santoni et al. reported cortical bone trajectory (CBT) as a method of inserting pedicle screws to obtain more solid fixation, and proposed the use of cortical trajectory screws with a more closely placed thread (cortical screws) for CBT. Since the entry trajectory in CBT differs from that in the traditional trajectory, it is unclear whether the increased strength derives from the specific trajectory or the shape of the screw thread in contact with the cortical bone. Whether the use of cortical screws is always required with CBT thus remains unclear. The authors therefore investigated the relationship between screw entry trajectory and screw thread characteristics and pullout strength in animal experiments.
\end{abstract}

METHODS Lumbar vertebrae (L1-L4) from 4-month-old female pigs were randomly assigned to one of 4 groups, with cancellous screws or cortical screws inserted via the traditional trajectory or CBT. For pullout strength testing, the screw was pulled out vertically against the direction of insertion. Rod pullout testing (toggle testing) was also performed, and the peak breaking strength was measured.

RESULTS The maximum pullout strength was significantly greater for CBT using cortical screws than for the traditional trajectory using cancellous screws. Pullout strength tended to be higher when cortical screws were used in both CBT and the traditional trajectory, although the difference was not significant. Toggle testing showed no significant differences among the 4 groups.

CONCLUSIONS The specific unconventional trajectory seemed to have a major impact on the increased strength obtained with CBT.

http://thejns.org/doi/abs/10.3171/2014.9.SPINE1484

KEY WORDS cortical bone trajectory; screw thread; spinal fusion; pedicle screw; technique

$\mathrm{S}$ PINAL surgery has rapidly progressed in recent years, thanks to improvement in diagnostic techniques, including imaging modalities, as well as advances in surgical procedures and the development of new surgical materials. Such progress has greatly contributed to patients' early reintegration into society and improved quality of life. Transformation of spinal surgery over the past 10 years, particularly transformation of the techniques and speed of surgical methods, has been remarkable. Numerous systems have emerged, all with the same ultimate aim of ensuring solid fixation and fusion. Posterior fixation by means of pedicle screws is the mainstay of surgical instru- mentation currently in use for patients with degenerative disorders of the lumbar spine. The pedicle screws used for fixation are generally inserted along the axis of the pedicle of the lumbar arch of the vertebral body. When this entry route is used, the screw does not come into contact with the cortical bone of the pedicle of the vertebral arch, but is inserted into the cancellous bone tissue in the pedicle and vertebral body. This means that screw fixation is achieved through insertion into cancellous bone. However, in elderly patients and others with osteoporosis, resorption of cancellous bone trabeculae may present problems with screw fixation, leading to development of such complica-

ABBREVIATIONS CBT = cortical bone trajectory; $\mathrm{PMMA}=$ polymethylmethacrylate; $\mathrm{TT}=$ traditional trajectory . SUBMITTED February 4, 2014. ACCEPTED September 19, 2014.

INCLUDE WHEN CITING Published online January 16, 2015; DOI: 10.3171/2014.9.SPINE1484.

DISCLOSURE The authors report no conflict of interest concerning the materials or methods used in this study or the findings reported in this paper. 
tions as loosening and displacement of screws., ${ }^{2,3,5,6} \mathrm{~A}$ variety of techniques have been developed to address these problems, including the combined use of hooks and sublaminar wiring, and the use of hydroxyapatite sticks to fill pedicle screw holes.

In 2009, Santoni et al. reported cortical bone trajectory (CBT) as a method of inserting pedicle screws to obtain more solid fixation. ${ }^{4}$ CBT was reported to be a novel entry trajectory for pedicle screws to maximize the area of contact with cortical bone and provide, in biomechanical tests, strength equivalent to or greater than that achieved by the traditional pedicle screw method.

The traditional pedicle screw method uses a screw designed for insertion in cancellous bone (cancellous screw), whereas the cortical trajectory screw (cortical screw), which maximizes thread contact with the higher density cortical bone, was proposed for $\mathrm{CBT}^{4}$ In general, cortical screws used in orthopedic surgery have a dense thread with a smaller bite (difference between the outer and root diameters), whereas screws used for cancellous bone have a larger bite and a larger pitch (distance between threads). ${ }^{1}$ Since the entry trajectory in CBT differs from that in the traditional trajectory, which is made along the axis of the pedicle of the vertebral arch, it is unclear whether the increased strength derives from the specific trajectory or the characteristics of the screw thread in contact with the cortical bone. Whether the use of cortical screws is always required with CBT thus remains unclear. We therefore investigated the relationship between screw entry trajectory and screw shape in animal experiments, with the aim of clarifying the reasons for the increased strength with CBT.

\section{Methods}

Lumbar vertebrae of 4-month-old female LWD crossbred pigs were used for the experiment. After removal of the surrounding tissue, vertebrae were severed at the intervertebral level: 4 vertebrae from each animal were used for mechanical testing, and 1 vertebra was used for bone mineral density measurement. For mechanical testing, screw pullout testing was carried out on 48 vertebrae from 12 animals, and rod pullout strength testing on 36 vertebrae from 9 animals. Bone mineral density of the fifth lumbar vertebra from each animal was measured by microcomputed tomography (inspeXio SMX-90 CT, Shimadzu Corp.) with use of a phantom. Legacy screws (Medtronic Japan) designed for traditional trajectory use were used as cancellous screws, and Solera screws (Medtronic) designed for CBT were used as cortical screws (Fig. 1). Screws with fixed heads (length $25 \mathrm{~mm}$, diameter $4.5 \mathrm{~mm}$ ) were used. Fenestration of the insertion hole was performed using an awl, and after preparation of the trajectory using either a Lenke probe or a 3-mm-diameter drill as a power tool, the screws were inserted after tapping with a 4.5-mm-diameter drill. The spinous process, which interferes with entry of the screw, was removed for CBT. Four vertebrae from each animal were randomly assigned to one of 4 groups, with cancellous screws or cortical screws inserted via the traditional trajectory or CBT: the cortical CBT group, in which cortical screws were inserted via CBT; the cancellous CBT group, in which cancellous screws were inserted
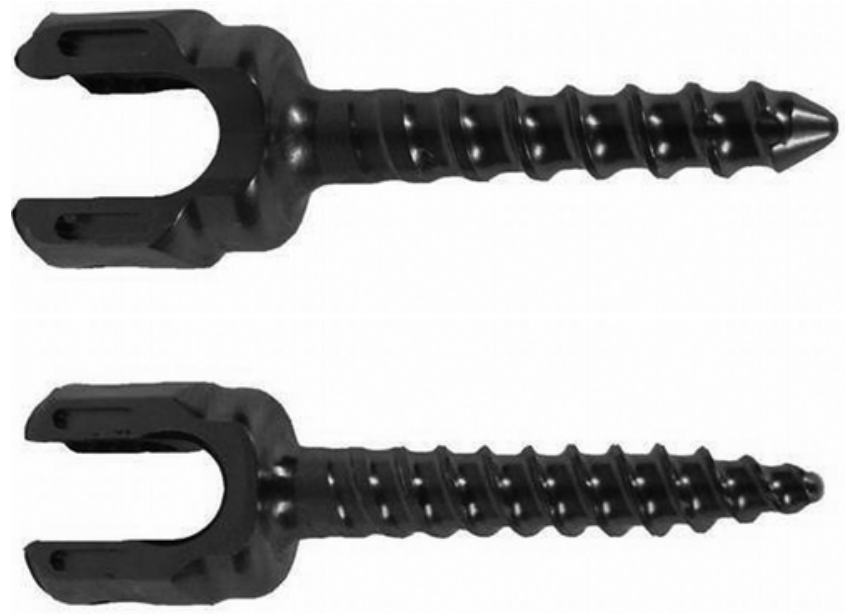

FIG. 1. Details of screws used in this study (length $25 \mathrm{~mm}$, diameter $4.5 \mathrm{~mm})$. Upper: Cancellous screw (Legacy, Medtronic)-outer diameter $4.59 \pm 0.79 \mathrm{~mm}$, root diameter $3.42 \pm 0.46 \mathrm{~mm}$, pitch $2.64 \pm 0.22$ $\mathrm{mm}$. Lower: Cortical screw (Solera, Medtronic)—outer diameter $4.54 \pm$ $0.84 \mathrm{~mm}$, root diameter $3.38 \pm 0.45 \mathrm{~mm}$, pitch $1.99 \pm 0.02 \mathrm{~mm}$.

via CBT; the cortical traditional trajectory (TT) group, in which cortical screws were inserted via the traditional trajectory; or the cancellous TT group, in which cancellous screws were inserted via the traditional trajectory.

After the screws were inserted, their positions were confirmed on radiographs (Fig. 2), and if penetration of cortical bone or breach of the pedicle was observed, all vertebrae from that animal were excluded. Screw pullout testing was finally performed on vertebrae from 9 animals and rod pullout testing on vertebrae from 6 animals. In screw pullout testing, the screw was connected to an insertion screwdriver and pulled out. Therefore, the connected area remained undamaged, and the screw could be reused. However, in rod pullout testing, the screws were damaged or the rods were deformed after a breaking test, because
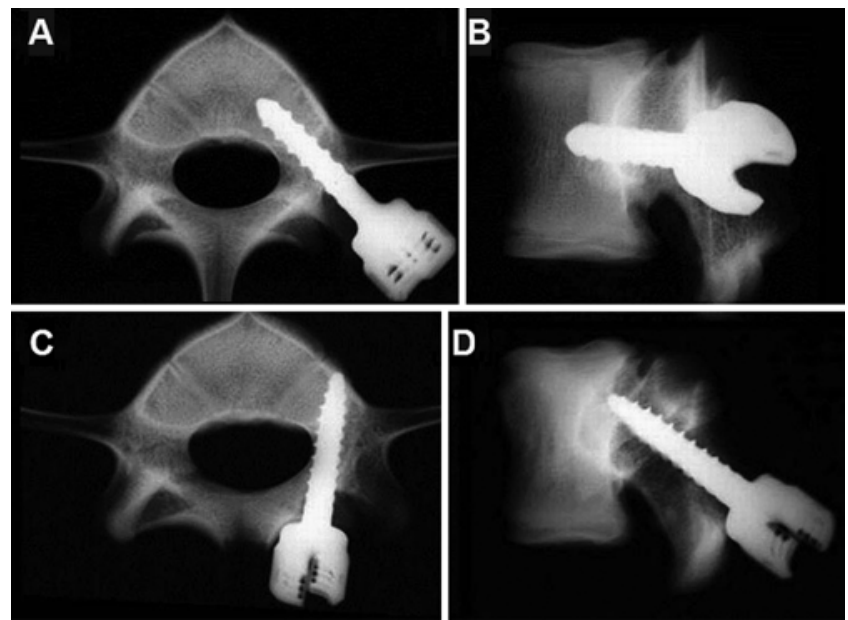

FIG. 2. Radiographs showing an axial view of the traditional trajectory (A), a lateral view of the traditional trajectory (B), an axial view of CBT (C), and a lateral view of CBT (D). Via the traditional trajectory, screws were inserted so that they were not in contact with cortical bone. Via CBT, screws were inserted so as to be in contact with cortical bone on the medial side of the pedicle of the vertebral arch and the craniolateral side of the pedicle of the vertebral arch. 
the screw was connected to the rod with a set screw and twisted off. Since a larger quantity of materials than expected was consumed, the decision was made to reduce the number of samples for economic reasons. Vertebrae in which screws had been inserted were adjusted so that the screws were placed vertically; the vertebrae were then embedded in blocks of polymethylmethacrylate (PMMA) resin. Vertical pullout strength testing and toggle strength testing were performed according to the methods described by Santoni et al. ${ }^{4} \mathrm{~A}$ universal testing machine, Instron 4467 (Instron Japan), was used for mechanical testing. For pullout strength testing, the screw was connected to an insertion screwdriver, and the vertebra in the PMMA block was fixed to the mechanical testing machine with a vise (Fig. 3). The screw was pulled out vertically against the direction of insertion at a speed of $10 \mathrm{~mm} /$ minute, and the peak breaking strength was measured. The slope of the approximately straight line of the linear region from the weight-versus-pullout-distance curve was defined as rigidity and measured. Following the methods of Santoni et al., a rod was attached to the screw to produce a combined screw and rod structure, and rod pullout testing (toggle testing) was performed. ${ }^{4}$ The screw was fixed vertically in the same way as in the pullout testing, and the rod was adjusted during screw insertion so that it ran parallel to the spinous process in the craniocaudal direction. The vertebra to which a rod was connected was fixed to the testing machine with a vise, and a special device was used to connect the caudal part of the rod to the testing machine (Fig. 4). The rod was connected at a position $40 \mathrm{~mm}$ from the screw and pulled upward at a speed of $10 \mathrm{~mm} / \mathrm{minute}$, and the peak breaking strength and rigidity were measured.

SPSS Statistics version 20 (IBM Japan) was used for statistical analysis. Two factors, trajectory and screw type, were analyzed by repeated 2-way analysis of variance, and a multiple comparison test (Tukey's test) was used to compare the 4 groups. Data are shown as mean \pm SD. A p value $<0.05$ was considered statistically significant.

\section{Results}

Bone mineral density is a specific value for an individual animal, and it is almost the same in every vertebra in
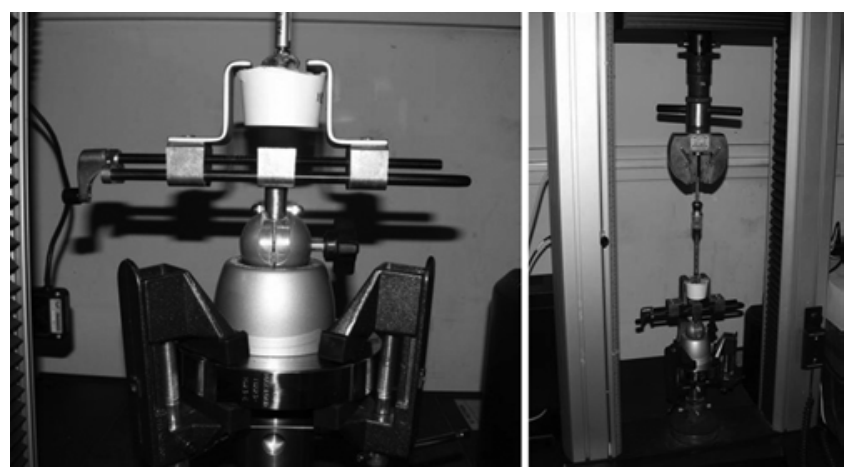

FIG. 3. Vertical pullout testing. The screw was connected to an insertion screwdriver, and the vertebral body was fixed in a PMMA block. Then, the vertebral body was fixed to a mechanical testing machine with a vise. The screw was pulled out vertically upward against the direction of insertion.
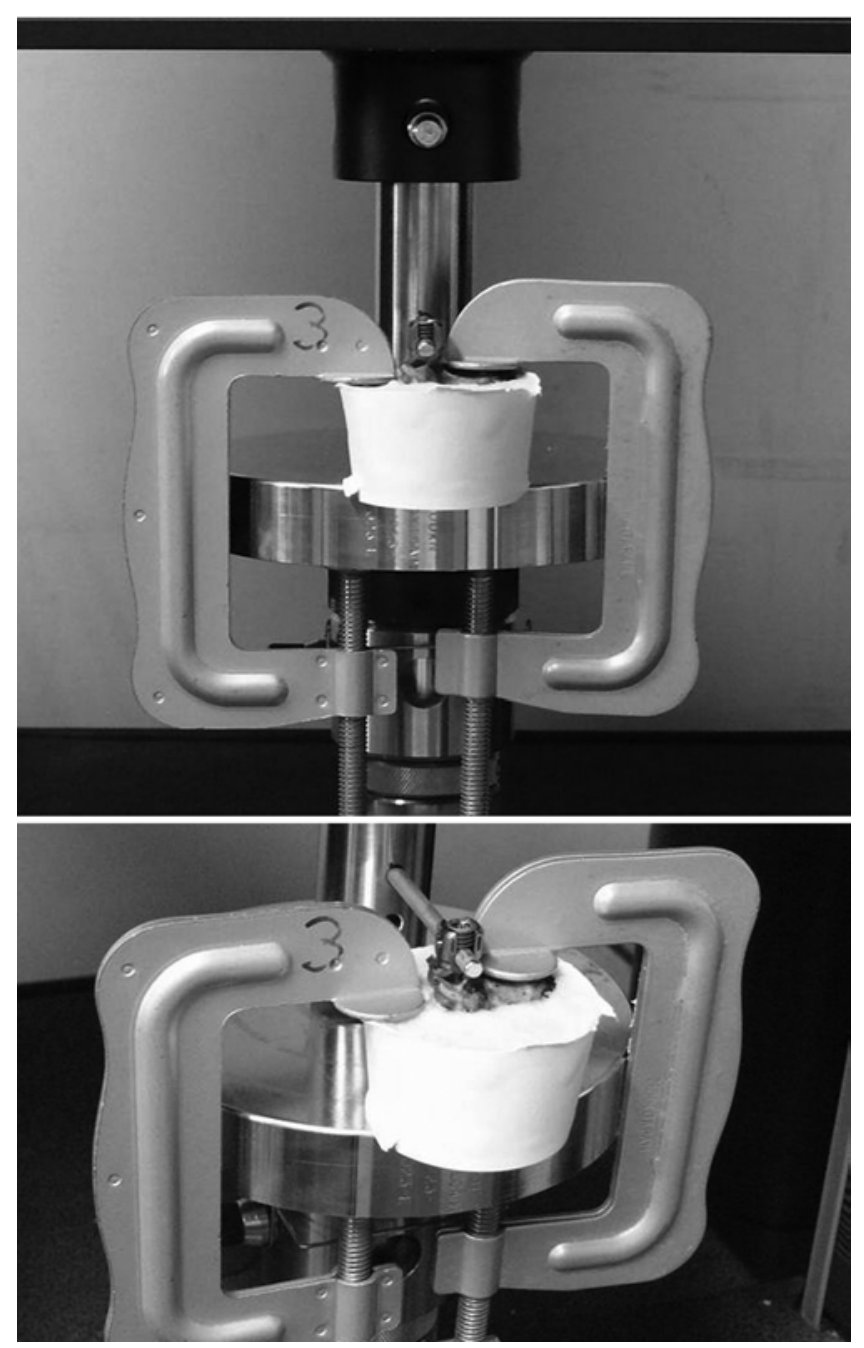

FIG. 4. Toggle testing. The structure, composed of a screw and a rod, was grasped by a special device, and pulled up parallel to the direction of screw insertion.

a single animal. In our study, we used the L-5 vertebra for the measurement of bone mineral density, and the L1-4 vertebrae, for the mechanical testing. In the total of 21 animals tested, 6 were observed to have 1 or more screws that penetrated or breached the vertebrae. For this reason, we excluded the entire data set of these 6 animals, and used the data from the remaining 15 animals, in which all of the screws could be successfully inserted, for screw pullout testing and rod pullout testing. The mean bone mineral density for the vertebrae from all 15 animals was $191.3 \pm$ $22.4 \mathrm{mg} / \mathrm{cm}^{3}$ (range $151.0-239.1 \mathrm{mg} / \mathrm{cm}^{3}$ ). Since the entire data sets of the animals with deficient data were excluded, it was presumed that bone mineral density had no effect on the accuracy of the results of the mechanical testing.

\section{Screw Pullout Testing}

The maximum pullout strength was $764.6 \pm 128.9 \mathrm{~N}$ in the cortical CBT group, $645.5 \pm 131.2 \mathrm{~N}$ in the cancellous CBT group, $718.3 \pm 114.2 \mathrm{~N}$ in the cortical TT group, and $590.1 \pm 80.5 \mathrm{~N}$ in the cancellous TT group (Fig. 5). Analysis of variance showed no significant difference 
between trajectories $(\mathrm{p}=0.196)$ but showed a significant difference between the 2 types of screws $(p=0.003)$. Multiple comparison testing showed a significant difference between the cortical CBT and cancellous TT groups ( $\mathrm{p}$ $=0.015)$. Rigidity was $314.2 \pm 74.6 \mathrm{~N} / \mathrm{mm}$ in the cortical CBT group, $295.6 \pm 55.5 \mathrm{~N} / \mathrm{mm}$ in the cancellous CBT group, $213.2 \pm 44.5 \mathrm{~N} / \mathrm{mm}$ in the cortical TT group, and $209.7 \pm 66.0 \mathrm{~N} / \mathrm{mm}$ in the cancellous TT group (Fig. 6). Analysis of variance showed no difference between the 2 types of screws inserted via the same trajectory $(p=0.616)$ but showed a significant difference between the two trajectories $(p=0.001)$. Multiple comparison testing showed significant differences between the cortical CBT group and the cortical TT and cancellous TT groups $(p=0.013, p$ $=0.010$ ). A significant difference was also shown between the cancellous CBT and cancellous TT groups $(\mathrm{p}=0.042)$.

\section{Rod Pullout Testing (Toggle Testing)}

The maximum pullout strength was $635.8 \pm 116.2 \mathrm{~N}$ in the cortical CBT group, 622.2 $\pm 61.1 \mathrm{~N}$ in the cancellous CBT group, $643.1 \pm 76.8 \mathrm{~N}$ in the cortical TT group, and $653.0 \pm 40.4 \mathrm{~N}$ in the cancellous TT group (Fig. 7). Analysis of variance showed no significant difference between either the trajectories or the types of screws $(\mathrm{p}=0.561, \mathrm{p}=$ 0.954). Multiple comparison testing showed no significant differences among the 4 groups. Rigidity was $127.1 \pm 42.9$ $\mathrm{N} / \mathrm{mm}$ in the cortical CBT group, $118.6 \pm 43.3 \mathrm{~N} / \mathrm{mm}$ in the cancellous CBT group, 127.2 $\pm 27.7 \mathrm{~N} / \mathrm{mm}$ in the cortical TT group, and $121.4 \pm 27.9 \mathrm{~N} / \mathrm{mm}$ in the cancellous TT group (Fig. 8). Analysis of variance showed no significant difference between either the trajectories or the types of screws $(p=0.922, p=0.638)$. Multiple comparison testing showed no significant difference among the 4 groups.

\section{Discussion}

CBT has been reported to provide approximately 1.3

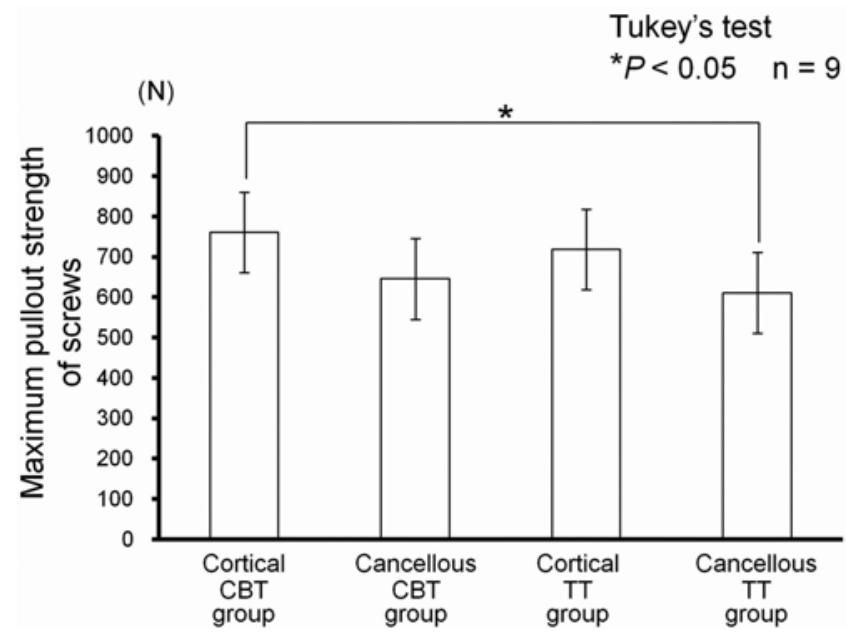

FIG. 5. Maximum pullout strength of screws during pullout testing. Pullout strength was significantly greater in the cortical CBT group than in the cancellous TT group. Cortical CBT group, cortical screws were inserted via CBT; cancellous CBT group, cancellous screws were inserted via CBT; cortical TT group, cortical screws were inserted via traditional trajectory; cancellous TT group, cancellous screws were inserted via traditional trajectory. Error bars indicate SDs.

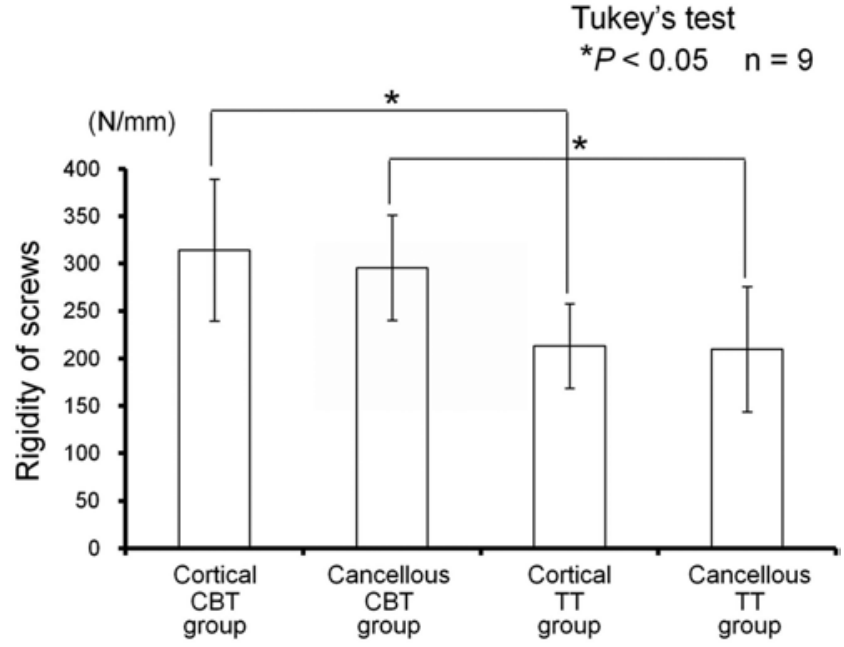

FIG. 6. Rigidity of screws during pullout testing. Rigidity was significantly higher in the cortical CBT group than in the cortical TT and cancellous TT groups, and in the cancellous CBT group than in the cancellous TT group. Error bars indicate SDs.

times greater pullout strength than the traditional trajectory in mechanical studies in cadavers, ${ }^{4}$ and its application is anticipated in patients with reduced bone quality due to osteoporosis and other conditions. The present study also showed that when cortical screws were inserted via CBT, the maximum pullout strength was approximately $29.5 \%$ higher than when cancellous bone screws were inserted via the traditional trajectory, representing a significant difference. Analysis of variance showed no difference between the two trajectories, although the pullout strength tended to be greater with CBT. A comparison between the types of screws showed that pullout strength increased significantly when cortical screws were used. Analysis of variance for rigidity similarly showed no difference between the two types of screws when inserted via the same trajectory, and a significant difference between trajectories irrespective of the type of screw, with pullout strength being greater with CBT. These results indicate that although both the screw thread characteristics and entry trajectory

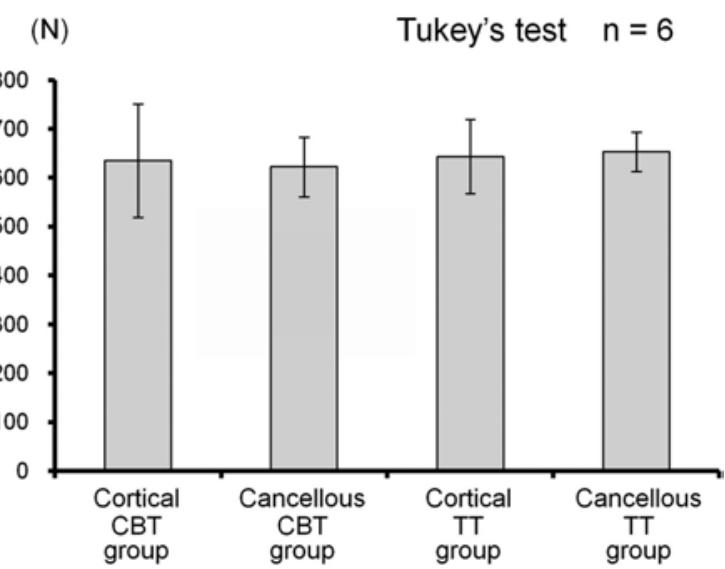

FIG. 7. Maximum pullout strength of rods during pullout testing. No significant difference was noted among the 4 groups. Error bars indicate SDs. 


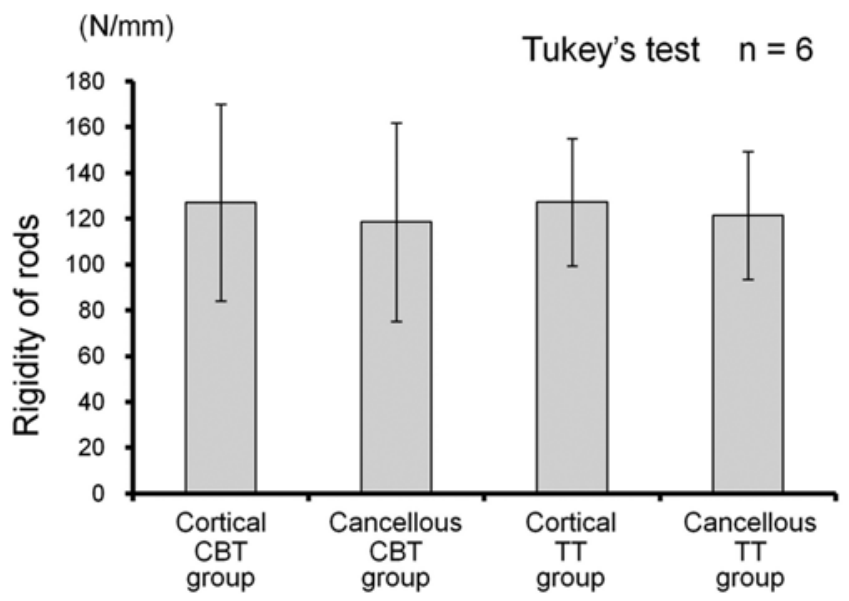

FIG. 8. Rigidity of rods during pullout testing. No significant difference was noted among 4 groups. Error bars indicate SDs.

contributed to the increased pullout strength obtained with CBT, the specific trajectory that enabled contact with cortical bone seemed to be the more influential factor. Our results also suggested that cortical screws provide more solid fixation via both CBT and the traditional trajectory, although the difference was not significant.

Of the two screw designs used in the present study, Solera has a tapered tip with almost the same outer and root diameters as those of Legacy. The pitch of Solera, however, is much denser. This can be regarded as the decisive difference between the two designs. In a narrow sense, controversy may exist as to the definitions of these two types of screws under the terms "cortical screw" and "cancellous screw." As far as we are aware, however, the terms "cortical screw" and "cancellous screw" have never been clearly defined. In our experiments, when the same two types of screws were inserted into two sites with obviously different rigidities, one type exhibited clearly greater pullout strength. This indicates that when screws are inserted into hard bones, the maximum strength cannot be obtained unless the appropriate design is adopted. It is a fact that of the two types of screws compared in our experiments, Solera is the more appropriate design for insertion via CBT, which maximizes the thread contact with cortical bone.

With the traditional trajectory, a screw has less contact with the cortical bone of the pedicle of the vertebral arch. It is inserted into the cancellous tissue in the pedicle of the vertebral arch, and the blunt end is fixed by the cancellous bone in the vertebral body. Therefore, under the assumption that cancellous bone is abundant, cancellous screws, which have a deeper cut and wider space, would be able to hold a larger bone volume between the threads, resulting in higher pullout strength, and, in fact, this screw is designed to meet this expectation. However, our study demonstrated that, regardless of the trajectory, cortical screws tended to have higher pullout strength. This may give the false impression that cortical screws can be used in any situation, and undermine the significance of cancellous screws. However, since cancellous bone is much less dense than cortical bone, the screw threads can cut their path by themselves when the screw is inserted in the bone, mean- ing that it can be inserted even without tapping. In addition, compared with a cortical screw, in which the threads are closely spaced, a cancellous screw can be inserted with a smaller number of rotations. These two characteristics give cancellous screws the advantage of a slightly shorter time for insertion. Further, when cancellous bone is abundant, it can be concluded that cortical screws may not always need to be used for a traditional trajectory, as seen in this study, because there is no evidence showing a significant difference in pullout strength between these two types of screws, even though cortical screws tended to show greater pullout strength. However, the number of elderly people is increasing. Bone density declines in the elderly, and problems can arise with pedicle screw fixation. Pedicle screw pullout strength is significantly lower in vertebral bodies with lower bone density, which may lead to early loosening and the development of pseudarthrosis. Some studies have even reported that it is impossible to obtain fixation sufficient to maintain the corrected position if the bone mineral density of vertebral bodies is severely reduced, and one publication has provided reference values calculated based on the insertion torque. However, since the pullout strength varies depending on the design of screws and the measurement method, it is difficult to determine a numerical value for the lower threshold to be used as a reference even for traditional screw entry and trajectories. What can be proposed is that it may be wise to select CBT and cortical screws when fixation is a concern. Since this study was conducted only to find out which thread type should be used for a trajectory with high rigidity such as a cortical bone trajectory, the kind of screws suitable for bone with reduced mineral density or for traditional trajectories was not investigated. However, CBT is considered to be suitable for patients without osteoporosis and younger patients, because CBT is also used for minimally invasive surgery. The ultimate goal of surgical fixation is solid fixation and fusion, and CBT would have only advantages and no disadvantages regarding increased fixation of screws even in patients without osteoporosis.

The reason for no difference in pullout strength during rod pullout testing was that, unlike screw pullout testing, in which traction was applied to the screw in an upward direction only, in rod pullout testing, the force was applied to the rod, meaning that a complex resultant force was generated that acted to pull the screw heads down in a caudal direction as well as in a vertical direction. The pullout strength might have been the same as that for the traditional trajectory, because effective fixation had not been performed at 3 points of the cortical bone; i.e., the entry site of CBT, the medial side of the pedicle of the vertebral arch, and the craniolateral side of the pedicle of the vertebral arch. This also suggests that the trajectory may be the major factor in the increased strength.

A few limitations of this study warrant mention. First, because the study comprised animal experiments, the data are not directly applicable to the human body. Pig bones are anatomically different from human bones. However, there would have been no point in performing this study using model bones, and very few institutions in Japan are legally authorized to use fresh cadavers. Therefore, pig lumbar vertebrae were regarded as the most appropriate 
for performing experiments on fresh, unfixed biomaterial. Next, to focus this study on investigation of the factors of trajectory and screw design alone, all screws used were of the same length and diameter. The effects of screw length and diameter (the proportion of the pedicle of the vertebral arch occupied by the screw) on pullout strength were thus not taken into account. Further studies are required to clarify the relationship between detailed screw position and strength, although these will require vertebrae from a large number of animals.

\section{Conclusions}

Maximum pullout strength was significantly greater for CBT using cortical screws compared with a traditional trajectory using cancellous screws. Pullout strength tended to be greater when cortical screws were used with both CBT and a traditional trajectory. The specific unconventional trajectory seemed to have a major impact on the increased strength obtained with CBT.

\section{References}

1. Asnis SE, Ernberg JJ, Bostrom MP, Wright TM, Harrington RM, Tencer A, et al: Cancellous bone screw thread design and holding power. J Orthop Trauma 10:462-469, 1996

2. Halvorson TL, Kelley LA, Thomas KA, Whitecloud TS III, Cook SD: Effects of bone mineral density on pedicle screw fixation. Spine (Phila Pa 1976) 19:2415-2420, 1994
3. Okuyama K, Sato K, Abe E, Inaba H, Shimada Y, Murai H: Stability of transpedicle screwing for the osteoporotic spine. An in vitro study of the mechanical stability. Spine (Phila Pa 1976) 18:2240-2245, 1993

4. Santoni BG, Hynes RA, McGilvray KC, Rodriguez-Canessa G, Lyons AS, Henson MA, et al: Cortical bone trajectory for lumbar pedicle screws. Spine J 9:366-373, 2009

5. Soshi S, Shiba R, Kondo H, Murota K: An experimental study on transpedicular screw fixation in relation to osteoporosis of the lumbar spine. Spine (Phila Pa 1976) 16:13351341,1991

6. Wittenberg RH, Shea M, Swartz DE, Lee KS, White AA III, Hayes WC: Importance of bone mineral density in instrumented spine fusions. Spine (Phila Pa 1976) 16:647-652, 1991

\section{Author Contributions}

Conception and design: Ueno. Acquisition of data: Ueno, Sakai, Tanaka, Imura. Analysis and interpretation of data: Ueno, Inoue, Uchida. Drafting the article: Tanaka. Critically revising the article: Ueno. Reviewed submitted version of manuscript: Saito, Nakazawa, Takahira, Mabuchi. Statistical analysis: Ueno. Study supervision: Takaso.

\section{Correspondence}

Masaki Ueno, Department of Orthopaedic Surgery, Machida Keisen Hospital, 1546-2, Ogawa, Machida, Tokyo 194-0003, Japan. email: masaki.ueno00@gmail.com. 